\title{
/CORRESPONDENCE
}

\section{Giving up on Taxol}

To the editor:

I was disturbed by a sentence in Lisa Conte's letter the the editor (Bio/Technology 11:765, July). She wrote, "Had we discovered taxol, given the scarcity of the Pacific yew tree, we would not have developed it." Taxol is a compound that has the possibility of significantly extending life and improving the quality of life for many cancer patients. It is not a trivial decision to abandon such a compound.

The development of taxol-or indeed almost any compound from slow growing plant species-does not need to threaten the producing species. For example, a number of academic and industrial laboratories have established plant cell cultures that produce significant levels of taxol.

I have heard other anecdotes about pharmaceutical companies dropping research on a promising compound because it was too complex for commercial chemical synthesis and came from slow growing and rare plant species. In these cases the existence

IMAGE
UNAVAILABLE
FOR COPYRIGHT
REASONS

\footnotetext{
"We appreciate your breakthrough work on $\mathrm{XJ}-305$, but the company prefers to market it."
} reduce dependence on extraction from natural sources by developing controllable processes using cell os cellular components. It is sad that a company would choose to allow a compound of potential significant benefit to humans be lost, when the tools of biotechnology could make it readily available.

Michael L. Shuler are inclined to underinsure their homes in flood plains. The application of psychometric measurements to technological risks, however, presents some unique methodological problems. Natural hazards like hurricanes, tornadoes, and floods are generally out of our control. But that is not how the public understands technology.

The gatekeepers and financial backers of a technology (or products thereof) may be honestly convinced of its safety. But the social response to technological risk is often a proxy for trust and control. And that is precisely why Dr. Miller's advice to those advancing new technologies or new products will not solve the problem of social acceptance. Public controversies over new technologies are society's way of asking for processing time: for sorting out values, for determining which evidence is trustworthy and which experts are credible, for ascertaining who speaks for the public interest and who speaks with a conflict of interest.

There was a time when government regulators were thought to be public gatekeepers to screen out harmful technology. But that perception has changed. Government review bodies are seen as mediating forces between industry and consumer activists. For the critical questions, the public and the media have come to depend upon citizen and science activists. There is of course emotional response to technological risk because emotion feeds public-spirited behavior; there is also financial response to technological risk because profit nourishes the behavior of the innovation sector. But it doesn'tend there. There is cultural rationality of risk that is distinguishable from technical rationality. You can find internal consistency in both approaches but they are driven by a different set of fundamental premises. For example, the popular culture may be skeptical about a technology without any established evidence of a risk to human health or the environment. Is that irrational? Were the French irrational for not accepting additives to their veal? Hardly. Perhaps the benefits were not worth the uncertainties.

Dr. Miller weighs heavily on the rationality of sciCornell University School of Engineering Ithaca, NY 14853

\section{Rational Risk Assessment}

To the editor:

In his Last Word, "Perception of Biotechnology Risks: The Emotional Dimension" (Bio/Technology 11:1075, September), Henry Miller has drawn attention to an important body of empirical studies on public perception of risk. The psychometric theory of risk perception, which has been under development for nearly two decades, is the major paradigm for these studies. Some of the earliest studies of public perception were directed at natural hazards. It is widely recognized that the public underestimates the probability and the adverse consequences of natural disasters. For example, people ence. The pursuit of pure science embodies the virtues of skepticism and patience. When nature is ready to reveal her secrets, we publish, otherwise we wait. We do not set a date to decide that a choice must be made between two competing hypotheses. Public policy cannot or will not wait for the ambiguities to disappear or for the incontrovertible evidence. We don't label science emotional when it gives its best shot under a deadline, under financial exigency, or under conditions of uncertainty. Paul Slovic, one of the world's leading experts on risk perception recently wrote: "psychometric research demonstrates that, whereas experts define risk in a narrow technical way, the public has a richer, more complex view that incorporates value-laden considerations such as equity, catastrophic potential, and controllability." Controversies over biotechnology should not be cast as struggles between rationality and emotion, but rather as struggles over different forms of rationality.

Sheldon Krimsky Department of Urban \& Environmental Policy

Tufts University Medford, MA 02155 